\title{
ON ASYMPTOTIC BEHAVIORS OF ANALYTIC MAPPINGS ON THE MARTIN BOUNDARY
}

\author{
MIKIO NIIMURA
}

ABSTRACT. Some generalizations of the analogue of Collingwood and Cartwright in the large of Iversen's theorem are given.

Let $f$ be a nonconstant analytic mapping of a hyperbolic Riemann surface $R$ into a Riemann surface $R^{\prime}$. Let $R^{*}$ and $R^{\prime *}$ denote the Martin compactification and any compactification of $R$ and $R^{\prime}$, respectively. $\Delta$ and $\Delta^{\prime}$ denote the Martin ideal boundary of $R$ and the ideal boundary of $R^{\prime}$, respectively. $\bar{A}, A^{C}$ and int $A$ mean the closure, the complement and the interior of a set $A\left(C R^{*}\right.$ or $\left.R^{\prime *}\right)$ with respect to $R^{*}$ or $R^{*}$, respectively. Let $\partial A$ denote the relative boundary of $A\left(C R\right.$ or $\left.R^{\prime}\right)$ with respect to $R$ or $R^{\prime}$ and $f_{G}$ the restriction of $f$ to $G(C R)$.

Let $\left\{G_{n}^{(e)}\right\}$ be a determinant sequence of Kerékjártó-Stoñlow's ideal boundary point $e$, and set $\Delta_{e}=\bigcap_{n} \overline{G_{n}^{(e)}}$ and $\Delta_{G_{n}^{(e)}}=\overline{G_{n}^{(e)}} \cap \Delta$. The cluster set of $f$ for $\Delta_{e}$ is defined by $C\left(f, \Delta_{e}\right)=\bigcap_{n} \frac{f\left(G_{n}^{(e)}\right)}{G^{n}}$, and the range of $f$ for $\Delta_{e}$ by $R\left(f, \Delta_{e}\right)=\bigcap_{n} f\left(G_{n}^{(e)}\right)$.

In this paper we assume that the harmonic measure of $\Delta_{e}$ is positive.

For $b \in \Delta_{1}$, let $F_{b}$ be a filter basis on $R$ with respect to the fine topology, and set $f^{\hat{}}(b)=\bigcap_{U \in F_{b}} \overline{f(U)}$. Here $\Delta_{1}$ denotes the set of minimal points in $\Delta$. If $f^{\wedge}(b)$ consists of a single point, then $f(b)$ is denoted by $\hat{f}(b)$.

We say that a curve $p=\lambda(t)(0 \leq t<1)$ on $R$ converges to $e$, when for every $n$, there exists $t(n)$ such that $\lambda(t) \subset G_{n}^{(e)}$ for all $t \geq t(n) . \bigcap_{t>0} \overline{\lambda(t)}$ denotes the end of this path: $p=\lambda(t)(0 \leq t<1)$. Let $\Gamma\left(f, \Delta_{G_{n}^{(e)}}\right)$ denote the set of asymptotic points along all the paths such that the end of each path is on $\Delta_{G_{n}^{(e)}}$, and set $\chi\left(f, \Delta_{e}\right)=\bigcap_{n} \Gamma\left(f, \Delta_{G_{n}^{(e)}}\right)$ and $\chi^{*}\left(f, \Delta_{e}\right)=$ $\bigcap_{n} \overline{\Gamma\left(f, \Delta_{G_{n}(e)}\right)^{n}}$. If for any neighborhood $V$ of $\alpha \epsilon R^{*}, V \cap \overline{\Gamma\left(f, \Delta_{G_{n}(e)}\right)}$ is a nonpolar set, we say $a \in \Gamma_{+}\left(f, \Delta_{G_{n}^{(e)}}\right)$ and set $\chi_{*}\left(f, \Delta_{e}\right)=\bigcap_{n} \Gamma_{+}\left(f, \Delta_{G_{n}^{(e)}}\right)$.

Lemma 1. If $a \in \chi_{*}\left(f, \Delta_{e}\right)^{c} \cap C\left(f, \Delta_{e}\right) \cap R^{\prime}$, then $a \in \operatorname{int} \overline{R\left(f, \Delta_{e}\right)}$.

Proof. Since a $\in \chi_{*}\left(f, \Delta_{e}\right)^{c} \cap R^{\prime}$, there exist a parametric disk $V$ about $\alpha$ and $G_{N}^{(e)}$ such that $V \cap \overline{\Gamma\left(f, \Delta_{G}(e)\right.}$ is a polar set. Let $w=\psi(q)$

Received by the editors October 2, 1974.

AMS (MOS) subject classifications (1970). Primary 30A72; Secondary 30A50.

Key words and phrases. Asymptotic point, Iversen's theorem, analytic mapping, compactification. 
( $q \in V$ ) be a local parameter of $V$, and we set $\psi(V)=\{w ;|w|<1\}(\psi(\alpha)=0)$, $W_{r}=\{w ;|w|<r, 0<r<1\}, C_{r}=\partial W_{r}$ and $\psi \circ f_{G}(e)=g$.

Since $\left.W_{1} \cap \overline{\Gamma\left(g, \Delta_{G}(e)\right.}\right)$ is a polar set, its linear measure is zero. Hence $\Gamma\left(g, \Delta_{G}(e) \cap C_{r}=\varnothing\right.$ for almost all $r$ in $0<r<1$. Let $C_{r}$ have this property and fix $r$.

Since a $\in C\left(f, \Delta_{e}\right)$, we see that $g^{-1}\left(W_{r}\right) \cap G_{n}^{(e)} \neq \varnothing$ for all $n \geq N$.

If there exists $G \in\left\{G_{n}^{(e)}\right\}$ such that $G \subset G_{N}^{(e)}$ and $g(G) \subset W_{r}$, then for each point $b$ of a set $H_{e}\left(\subset \Delta_{1} \cap \Delta_{e}\right)$ whose harmonic measure is positive, $\hat{g}_{G}(b) \in \bar{W}_{r}$. Indeed the harmonic measure of $\Delta_{e}$ is positive and $g_{G}$ is a Fatou mapping of $G$ into $W_{r}$.

Hence there exists an asymptotic path $\gamma$ from a point of $G$ to each point $b$ of $H_{e}$ such that $\lim _{p \rightarrow b ; p \in \gamma} g_{G}(p)=\hat{g}_{G}(b)$. On the other hand, since $\bar{W}_{r} \cap$ $\Gamma\left(g, \Delta_{G}\right)\left(\supset \hat{g}_{G}(b)\right.$, for $\left.b \in H_{e}\right)$ is a polar set, the harmonic measure of $H_{e}{ }_{e}$ is zero. This is a contradiction. Thus for all $n \geq N$, we conclude that $G_{n}^{(e)}$ $\cap \partial g^{-1}\left(W_{r}\right) \neq \varnothing$.

If $\partial g^{-1}\left(W_{r}\right)$ contains closed Jordan curves accumulating to $e$, then we see easily that $w \in R\left(g, \Delta_{e}\right)$ for any point $w$ on $C_{r}$.

If for all $n \geq N, G_{N}^{(e)}$ has at least one noncompact $\gamma_{n}$ of $\partial g^{-1}\left(W_{r}\right)$, let $z=\phi(p)$ be a local parameter about $p \in G_{N}^{(e)}$, and set $h=g \circ \phi^{-1}$. A func. tion element $Q(w)$ of $z=h^{-1}(w)$ can be continued analytically along $C_{r}$ infinitely often. Indeed if not, when $w$ tends to a point $w_{1}\left(\in C_{r}\right)$ along $C_{r}$, $\gamma_{n}$ is a path whose end is on $\Delta_{G_{n}^{(e)}}$, and so $w_{1} \in \Gamma\left(g, \Delta_{G_{n}^{(e)}}\right)$. This is a contradiction.

Therefore any point $w$ on $C_{r}$ corresponds to an infinite number of points on $\gamma_{n}$ for any $n$, and hence $w \in R\left(g, \Delta_{e}\right)$.

Therefore since for any point $p$ of $W_{1}$, any neighborhood $\left(C W_{1}\right)$ of $p$ contains points of $R\left(g, \Delta_{e}\right)$, we get $W_{1} \subset \overline{R\left(g, \Delta_{e}\right)}$ and $\alpha \in \operatorname{int} \overline{R\left(f, \Delta_{e}\right)}$, as claimed.

Corollary 1. If $C\left(f, \Delta_{e}\right)$ is nowhere dense, then $C\left(f, \Delta_{e}\right) \cap R^{\prime} \subset \chi_{*}\left(f, \Delta_{e}\right)$.

Proof. If $\alpha \in$ int $\overline{R\left(f, \Delta_{e}\right)}$, for a neighborhood $V$ of $\alpha$, any neighborhood $(C V)$ of any point $\beta \in V$ contains at least one point of $R\left(f, \Delta_{e}\right)$ and $C\left(f, \Delta_{e}\right)$ is not nowhere dense. Thus we have $C\left(f, \Delta_{e}\right) \cap R^{\prime} \subset \chi_{*}\left(f, \Delta_{e}\right)$.

Lemma 2. If $a \in \chi_{*}\left(f, \Delta_{e}\right)^{c} \cap \chi\left(f, \Delta_{e}\right)^{c} \cap C\left(f, \Delta_{e}\right) \cap R^{\prime}$, then $a \in R\left(f, \Delta_{e}\right)$.

Proof. Suppose that $a \notin R\left(f, \Delta_{e}\right)$.

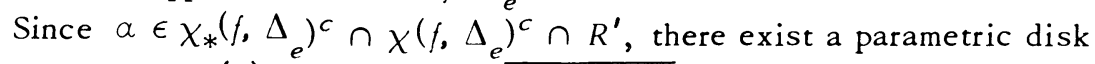
$U$ about $\alpha$ and $G_{N}^{(e)}$ such that $U \cap \overline{\Gamma\left(f, \Delta_{G}(e)\right.}$ is a polar set and $\alpha \notin$ $\Gamma\left(f, \Delta_{G N}^{(e)}\right)$.

All the a.points of $f_{G}(e)$ are contained in a finite set of parametric 
disks $\left\{U_{k}\right\}(k=1,2, \ldots, L)$ such that $U_{i} \cap U_{j}=\varnothing(i \neq j)$. Let $V$ be a parametric disk about a satisfying $V \subset\left(\bigcap_{k=1}^{L} f_{G}^{(e)}\left(U_{k}\right)\right) \cap U$. We fix $r$ such that $\Gamma\left(g, \Delta_{G}(e)\right) \cap C_{r}=\varnothing$. There exists a diameter $d_{r}$ of $W_{r}$ such that $\Gamma\left(f_{0} \Delta_{G}(e)\right) \cap d_{r}=\varnothing$. There exists a diameter $d_{r}$ of $W_{r}$ such that $\Gamma\left(f, \Delta_{G_{N}}(e)\right) \cap d_{r}=\varnothing$.

Since $b \in R\left(f, \Delta_{e}\right)$ for $b \in C_{r}$, there exists a connected component $D$ of $g^{-1}\left(W_{r}\right)$ which is not relatively compact. Choose a point $p$ on $\partial D$ which is mapped by $g$ to an endpoint of $d_{r}$. The function element $Q(w)$ corresponding to $p$ can be continued analytically along $d_{r}$ through the point 0 to the antipodal point and $d_{r}$ is mapped on a cross-cut of $D$. But on the other hand, since $D$ does not contain the zeros of $g$, we have a contradiction, and we conclude that $a \in R\left(f, \Delta_{e}\right)$.

Theorem 1. If $R^{\prime *}$ is a metrizable and resolutive compactification of $R^{\prime}$ and, for at least one $n, \overline{\Gamma\left(f, \Delta_{G_{n}}^{(e)}\right)}$ is a polar set, then $R\left(f, \Delta_{e}\right)^{c} \cap R^{\prime} \subset$ $\chi\left(f, \Delta_{e}\right)$.

Proof. From Lemma 2, we have $R\left(f, \Delta_{e}\right)^{c} \cap R^{\prime} \subset \chi_{*}\left(f, \Delta_{e}\right) \cup \chi\left(f, \Delta_{e}\right)$ $\cup C\left(f, \Delta_{e}\right)^{c}$.

If $C\left(f, \Delta_{e}\right)^{c} \neq \varnothing$, there exist a parametric disk $V$ and $G \in\left\{G_{n}^{(e)}\right\}$ ( $G \subset$ $\left.G_{n}^{(e)}\right)$ such that $f(G) \cap \bar{V}=\varnothing$. Since the mapping $f_{G}$ of $G$ into $R^{\prime}-\bar{V}$ is a Fatou mapping, it contradicts that the harmonic measure of $H_{e}$ is positive, as we see from the proof of Lemma 1.

Thus from $\Gamma_{+}\left(f, \Delta_{G_{n}^{(e)}}\right)=\varnothing$, we have $R\left(f, \Delta_{e}\right)^{c} \cap R^{\prime} \subset \chi\left(f, \Delta_{e}\right)$.

Lemma 3. If $\alpha \in \chi^{*}\left(f, \Delta_{e}\right)^{c} \cap C\left(f, \Delta_{e}\right) \cap R^{\prime}$, then $\alpha \in \operatorname{int} R\left(f, \Delta_{e}\right)$.

Proof. In Lemma 1, take "all $r$ in $0<r<1$ " instead of "almost all $r$ in $0<r<1$ " and consider " $W_{1} \cap \Gamma\left(g, \Delta_{G}(e)=\varnothing\right.$ " instead of " $W_{1} \cap$ $\overline{\Gamma\left(g, \Delta_{G(e)}\right)}$ is a polar set", then we have $w \in R\left(g, \Delta_{e}\right)$ for all $w: 0<|w|<1$ as in the proof of Lemma 1.

If $w_{0} \in W_{r / 2}\left(w_{0} \neq 0\right)$, we have $w_{0} \in C\left(g, \Delta_{e}\right)$ and $W_{r / 2}^{\prime} \cap \Gamma\left(g, \Delta_{G(e)}\right)$ $=\varnothing\left(W_{r / 2}^{\prime}=\left\{w ;\left|w-w_{0}\right|<r / 2\right\}\right)$, and hence $0 \in R\left(g, \Delta_{e}\right)$.

Thus we have $W_{1} \subset R\left(g, \Delta_{e}\right)$ and $a \in$ int $R\left(f, \Delta_{e}\right)$.

Theorem 2. $\overline{R\left(f, \Delta_{e}\right)^{c}} \cap C\left(f, \Delta_{e}\right) \cap R^{\prime} \subset \chi^{*}\left(f, \Delta_{e}\right)$.

Proof. From Lemma 3, we have

$$
X^{*}\left(f, \Delta_{e}\right)^{c} \subset C\left(f, \Lambda_{e}\right)^{c} \cup R^{\prime c} \cup\left(\text { int } R\left(f, \Delta_{e}\right)\right)
$$

that is, 


$$
\overline{R(f,} \overline{\left.1_{e}\right)^{c}} \cap C\left(f, \Delta_{e}\right) \cap R^{\prime} \subset \chi^{*}\left(f, \Delta_{e}\right) .
$$

Lemma 4. int $C\left(f, \Delta_{e}\right) \subset \overline{R\left(f, \Delta_{e}\right)}$.

Proof. If $a \in$ int $C\left(f, \Delta_{e}\right)$, for any neighborhood $U$ of $a$, there exists a parametric disk $V_{0}$ about $a_{0}$ satisfying $\overline{V_{0}} \subset U \cap C\left(f, \Delta_{e}\right)$. Since $a_{0} \epsilon$ $C\left(f, \Delta_{e}\right)$, there exists $p_{1} \in G_{1}^{(e)}$ such that $a_{1}=f\left(p_{1}\right) \in V_{0}$. We can take a parametric disk $V_{1}$ about $\alpha_{1}$ satisfying $\bar{V}_{1} \subset V_{0} \cap f\left(G_{1}^{(e)}\right)$. Repeating the same method, we have a sequence of parametric disks $\left\{V_{n}\right\}(n=1,2,3, \ldots)$ such that $\overline{V_{n+1}} \subset V_{n}$ and $\bar{V}_{n} \subset f\left(G_{n}^{(e)}\right) . \beta \in \bigcap_{n} \bar{V}_{n}$ is assumed by $f$ in any $G_{n}^{(e)}$, and hence $\alpha \in \overline{R\left(f, \Delta_{e}\right)}$.

Corollary 2. $R\left(f, \Delta_{e}\right)^{c} \cap R^{\prime} \subset \chi^{*}\left(f, \Delta_{e}\right)$ if and only if $\left.\overline{R\left(f, \Delta_{e}\right.}\right)=R^{\prime *}$.

Proof. If $C\left(f, \Delta_{e}\right) \neq R^{\prime *}$, there exists $\alpha_{0}$ such that $\alpha_{0} \in C\left(f, \Delta_{e}\right)^{c} \cap$ $R^{\prime} \subset R\left(f, \Delta_{e}\right)^{c} \cap R^{\prime}$. If $\alpha \in \chi^{*}\left(f, \Delta_{e}\right)$, then we have $a \in \overline{\Gamma\left(f, \Delta_{G_{n}(e)}\right)}$ for any $n$ and $0 \in \overline{\Gamma\left(g, \Delta_{G_{n}^{(e)}}\right)}$ for a parametric disk $V$ about $\alpha$. Since there exists $w_{n} \in W_{1 / n} \cap \Gamma\left(g, \Delta_{G_{n}^{(e)}}\right)$, there exists $p_{n} \in G_{n}^{(e)}$ such that $g\left(p_{n}\right) \in W_{1 / n}$. Since $p_{n}$ converges to $e$ and $g\left(p_{n}\right)$ converges to 0 , we see that $0 \in C\left(g, \Delta_{e}\right)$ and $\alpha \in C\left(f, \Delta_{e}\right)$. Hence we have $\chi^{*}\left(f, \Delta_{e}\right) \subset C\left(f, \Delta_{e}\right)$ and $\alpha_{0} \notin \chi^{*}\left(f, \Delta_{e}\right)$. Thus if $R\left(f, \Delta_{e}\right)^{c} \cap R^{\prime} \subset \chi^{*}\left(f, \Delta_{e}\right)$, from Lemma $\left.4, \overline{R\left(f, \Delta_{e}\right.}\right)=R^{\prime *}$.

Conversely if $\overline{R\left(f, \Delta_{e}\right)}=R^{\prime *}$, then we have, from Theorem 2,

$$
R\left(f, \Lambda_{e}\right)^{c} \cap R^{\prime}=R\left(f, \Lambda_{e}\right)^{c} \cap C\left(f, \Lambda_{e}\right) \cap R^{\prime} \subset x^{*}\left(f, \Lambda_{e}\right) .
$$

Corollary 3. If the characteristic function of $f(c f .[3])$ is unbounded, then $R\left(f, \Delta_{e}\right)^{c} \cap R^{\prime} \subset \chi^{*}\left(f, \Delta_{e}\right)$.

Proof. If $C\left(f, \Delta_{e}\right) \neq R^{\prime *}$, since $f_{G}$ is a Lindelöf mapping, as in the proof of Theorem 1, the characteristic function of $f$ is bounded, and a contradiction. Thus from Lemma 4 and Corollary 2 we get $R\left(f, \Delta_{e}\right)^{c} \cap R^{\prime} \subset$ $\chi^{*}\left(f, \Delta_{e}\right)$.

\section{REFERENCES}

1. E. F. Collingwood and M. L. Cartwright, Boundary theorems for a function meromorphic in the unit circle, Acta Math. 87 (1952), 83-146. MR 14, 260.

2. C. Constantinescu and A. Cornea, Ideale Ränder Riemannscher Flächen, Ergebnisse der Mathematik und ihrer Grenzgebiete, N. F., Band 32, Springer-Verlag, Berlin, 1963. MR 28 \#3151.

3. M. Heins, Lindelöfian maps, Ann. of Math (2) 62 (1955), 418-446. MR 17, 726.

4. K. Noshiro, On the theory of the cluster sets of analytic functions, Fac. Sci. Hokkaido Imperial Univ. 6 (1937), 217-231.

DEPARTMENT OF MATHEMATICS, SHIBAURA INSTITUTE OF TECHNOLOGY, 3-9-14, SHIBAURA, MINATO-KU, TOKYO, JAPAN 